\title{
Smart micro-sensoring: antibodies and aptamer-based micro-ELISA as performing offline/on line tool for allergens and mycotoxins detection in foods
}

\author{
L. Boschis ${ }^{1}$, N. Ciprianetti ${ }^{1}$, Elena Guli ${ }^{1}$, D. Spadaro ${ }^{2}$, M.L. Gullino ${ }^{2}$, R. Rinaldi ${ }^{3}$, C. Garino ${ }^{4}$, M. Arlorio \\ ${ }^{1}$ Trustech s.r.l., Torino, Italy \\ ${ }^{2}$ AGROINNOVA and Dept. Agricultural, Forestry and Food Sciences (DISAFA), University of Torino, \\ Torino, Italy \\ ${ }^{3}$ aizoOn Consulting Technology, Torino, Italy \\ ${ }^{4}$ Dipartimento di Scienze del Farmaco \& Drug and Food Biotechnology Center, Università del Piemonte \\ Orientale A. Avogadro, Novara, Italy
}

\begin{abstract}
Concerning food safety, the interest for smart and portable biosensor is growing. On the other side, current commercial solution still does not reach performance comparable to laboratory test. Micro-ELISA is a smart portable biosensor useful in implementing the paradigms of the Process Analytical Technology (PAT) to the food industry. The use of this biosensor device (Lab-On-a-Chip disposable card, LOC) is a promising tool devoted to the detection of biotic and abiotic contaminant during the whole food supply chain with the accuracy and precision of laboratory methods. Micro-ELISA probes are based on affinity biomolecules, both antibodies and aptamers can be used. Some examples of applications - obtained from the project "Food Digital Monitoring", supported by structural and National funds for Regional Development - are reported: lysozyme as food allergen and aflatoxin $B 1$ as food contaminant.
\end{abstract}

Keywords: Lab on Chip; microfluidic; Point of Care; ELISA

\section{INTRODUCTION}

Food safety is a top-flight issue involving the whole production chain, from crops and cattle breeding to processing facilities. Foodborne disease, indeed, is currently a critical public health concern worldwide. Being able to accurately assess the presence of contaminants, pathogens or allergens in foods is crucial, and there is a constant research for new performing analytical methods and data management tools.

Quality by Design (QbD) is simultaneously the new leading paradigm in the food industry and is directly inspired by the Process Analytical Technology (PAT) concept, originally introduced for the pharmaceutical industry in 2004 by the United States Food and Drug Administration [1-3]. This paradigm bases its shift on the hypothesis that the quality of the (food) products can and should be incorporated by process design and not by postproduction quality testing. Food industry faces indeed similar strict regulatory demands in terms of quality control, safety and traceability as well as the production of pharmaceuticals.
Currently, food safety is assessed by standard techniques, such as ELISA, chromatographic or mass-based analyses, carried out in central laboratories. This approach is not time-effective and exhaustive, because only a limited number of representative samples is tested. The development of point-ofcare-tests (POCT) could allow an efficient food quality management, by setting up local extensive monitoring programs and rapid screenings. This requires high sensibility, high selectivity, rapid and reliable biosensors, integrated with an automated control system. By coupling real-time or nearreal-time process monitoring capabilities with advanced sensors, the industry is gradually moving from inferential monitoring toward continuous measurement of core quality parameters. Research based development and implementation of PAT in the food industry is focused on improved control, rapid final product quality evaluation and increased productivity. A positive side effect can be industrial innovation and enhanced competitiveness. In fact, the interest towards "smart" analytical approaches based on the use of micro and nanosensors is continuously growing worldwide.

Portable and automated devices for the detection and characterization of biotic and abiotic contaminant during the whole food supply chain are pivotal in this context, and constitute a dynamic area in food processing, which is experiencing important developments coming mainly from the standpoint of food safety. Several sensing systems are described in the literature. Commonly they are based on an optical or electrochemical transducer functionalized with a biological recognizing element (enzymes, cells or affinity molecules) [4,5]. Several new commercially available tests are issued every year, but no one performs all the requirements needed for food analysis protocols, and it is still difficult to satisfy both accuracy and on-field reliability.

A new analytic platform named Micro-ELISA has been developed by Trustech (www.trustech.it) and combines capillary data collection to elaboration during the entire food processing. The Micro-ELISA device is based on a 
microfluidic ready-to-use disposable card (Lab-on-a-Chip, LOC) and a small portable related reader. Micro-ELISA has been designed to be automated and highly integrated with the whole control system software of food smart factories. Some examples of application will be tested during the project "Food Digital Monitoring" (FDM), funded by the Piedmont Region with European Funds for Regional Development. The project aims at developing a new methodology for the online and offline control of food processing based on the PAT paradigm. Following this vision, new portable and connected biosensors like Micro-ELISA are very important to monitor chemical, physical and microbiological parameters, in addition to data and information collected during the whole food production chain, which will allow the capillary control of industry production processes. This centralized monitoring system, indicated in the FDM project with the term "Dashboard" (or "Smart Dashboard"), will allow food companies to control the entire production and distribution chain by detecting occurring anomalies through diagrams that will quicken the identification of the problem. The dashboard will therefore enable the real time and near real time intervention directly in the production stages, thus improving the efficiency and limiting the random variability, the rebalancing and the product losses. Tools like Micro-ELISA are crucial to empower this concept of "Smart Factory".

In this work, the Micro-ELISA smart sensor is described, and the collected experimental data regarding food allergens and mycotoxins detection are shown. Micro-ELISA is a microfluidic point-of-care biosensor able to detect biotic and abiotic contaminants (e.g. allergens and mycotoxins) during the food supply chain. Micro-ELISA probes are based on affinity biomolecules, both antibodies and aptamers can be used. In this paper, two significant case studies are reported: lysozyme as food allergen and aflatoxin B1 as food contaminant.

Lysozyme, as gluten and lactose, entails allergy related risks in susceptible individuals. It is naturally present in eggs and egg derivates, and is often added as preservative in various foods (such as cheese and wine), medications and vaccines. The residues of this protein could represent a serious risk for allergic subjects. According to the EU Directive on labelling (2003/89/EU), if one or more of the ingredients listed in Annex IIIa of Directive 2000/13/EC (and following amendments) are present in wine, they must be indicated on the labelling of the product. Egg proteins like lysozyme used during the production need to be present at a detection level lower than $0.25 \mathrm{mg} / \mathrm{L}$.

Aflatoxins are hepatotoxic and carcinogenic secondary metabolic products, synthetized by fungi belonging in particular to the Aspergillus flavus and A. parasiticus species [6,7]. Aflatoxins are found as natural contaminants in many feedstuffs of plant origin, especially in cereals but also in fruits, hazelnuts, almonds and their products, intended for human or animal consumption [8]. It is well known that AB1 can cause chronic diseases in humans and animals, and can determine several adverse effects, such as hepatotoxicity, genotoxicity and immunotoxicity [9]. European legislation sets a maximum permitted concentration in human food (2-12 $\mu \mathrm{g} / \mathrm{kg})$, infant foods $(0.1 \mu \mathrm{g} / \mathrm{kg})$ and animal feeds $(5-50 \mu \mathrm{g} / \mathrm{kg})$ [9].

\section{SYSTEM OVERVIEW AND DESCRIPTION OF THE ANALYTICAL APPROACH}

In this work, a portable and automated diagnostic platform able to perform miniaturized ELISA assays has been set up, developed and applied to some case studies. The system is suitable for Point of Care (POC) analysis in agrifood quality control applications, and can be a remote node or a larger network connected to the control software of a "Smart Factory".

The Micro-ELISA device is composed by:

- disposable ready-to-use LOC cards;

- a light and automated reader.

The LOC has been designed to perform both direct, indirect, competitive and non-competitive assays based on antibodies as well as aptamers. The microfluidic architecture is made of microchambers devoted to reagent reservoirs, reaction chambers, optical detection areas and waste collection chambers, all connected through microchannels, as described in figure 1 .

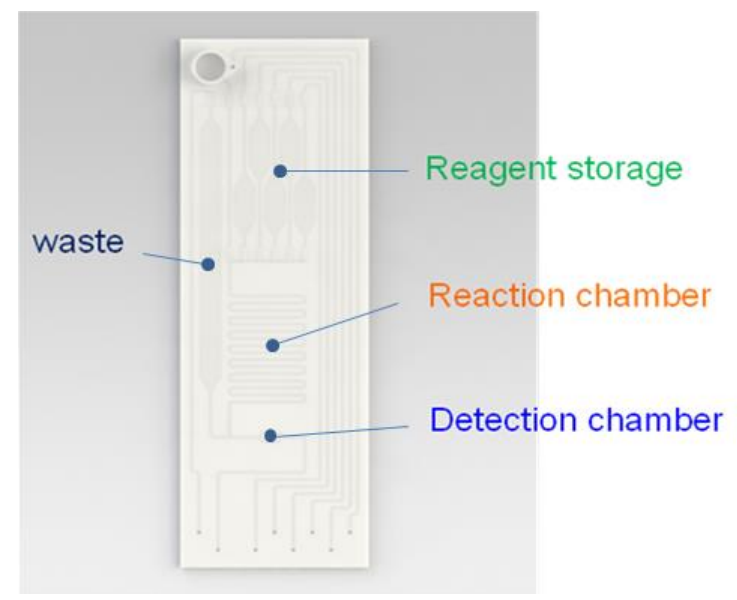

Fig.1. Microfluidic architecture of Micro-ELISA LOC.

Four analytical lines are present in the LOC architecture, in order to perform four tests in parallel. This configuration allows choosing between a semi-quantitative multi target measurement, being able to perform either four different analysis in parallel, or a single quantitative target measurement, being able to perform a calibration curve and one target quantitative analysis at the same time.

To perform a calibration curve at the same time of the analysis enables the accurate quantification of the target, and this is a main advantage of Micro-ELISA, that allows an accurate and precise detection even outside the lab. Most of currently available screening devices do not give this possibility, thus lacking in accuracy and precision, especially for low concentrated targets like aflatoxins. 
In this configuration, the first line is dedicated to the analyte analysis, while the second is dedicated to the blank (or max signal control, depending whether the ELISA is competitive or not), and the last two lines are measures of known reference standard analytes. The LOC, realized in polymethylmethacrylate (PMMA) and fabricated with micromilling and thermal bonding techniques, is designed to have all reagents preloaded, antibodies or aptamers are bound to the surface of the reaction region. In this configuration, the device is ready-to-use. The only operational step required is loading the sample and insert the LOC into the instrument, which can be done by either a user or a robotic system.

Once inserted in the instrument, the LOC card is automatically connected to pumps and electrovalves inside the reader, and the software can properly manage the loading of reagents. Appropriate ELISA protocols are executed automatically pumping reagents and samples through the microfluidic channels architecture. When the final reaction occurs, absorbance readings are performed. The software processes the data acquired by interpolating them with the calibration curve and calculating the concentration of the target analyte. Then, the result of analysis can be sent to the Smart Factory central control software.

The colorimetric detection was chosen as detection system. This is composed by a light source set at $450 \mathrm{~nm}$, a light emitting diode (LED), and a photodiode used as collector. Experimental tests have shown that the method is selective and very sensitive, reaching detection limits in the order of parts per million (ppt).

The method was tested for aflatoxin M1 in milk, B1 in corn and wheat, and to discover traces of allergens like lysozyme in wine.

The molecular probes used were antibodies, properly immobilized inside the reaction chambers.

As well known, the preservation of antibodies requires low temperatures, which limits their use outside the lab. Aptamers represent a stable and promising probe alternative in food biosensors [10]. As a matter of fact, aptamers are much more stable than antibodies, and do not require to be stored at low temperatures $[11,12]$. The aptamer-based LOC is currently under development, and experimental data will not be disclosed here. The employed detection system is based on fluorescence. As well as competitive and non-competitive ELISA, two different approaches have been designed. In the first one, fluorescent aptamers are bound to the surface of reaction chambers and a complementary oligo labelled with a quencher is added. When the target is absent, the fluorescent signal is switched off, while, in its presence, its binding with the aptamer is able to displace the quencher (Fig. 2). The fluorescence is then proportional to the concentration of the target molecules.

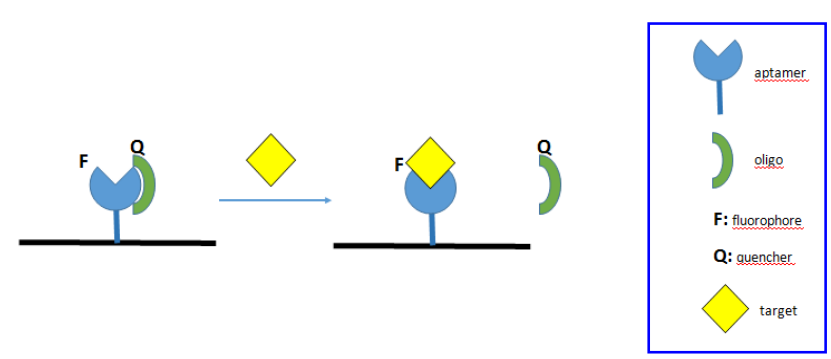

Fig. 2. Quenching oligo bound to the fluorescent aptamer immobilized on the surface of the reaction chamber is displaced by the arrival of the target.

In the second design, non-fluorescent aptamers are immobilized on the surface of the reaction chamber, and a fluorescently labelled complementary oligo is added. The arrival of the target removes the oligo, which accumulates in the waste. The drop of fluorescence in the detection chamber is then inversely proportional to the presence of the target molecules, as described in Fig. 3.

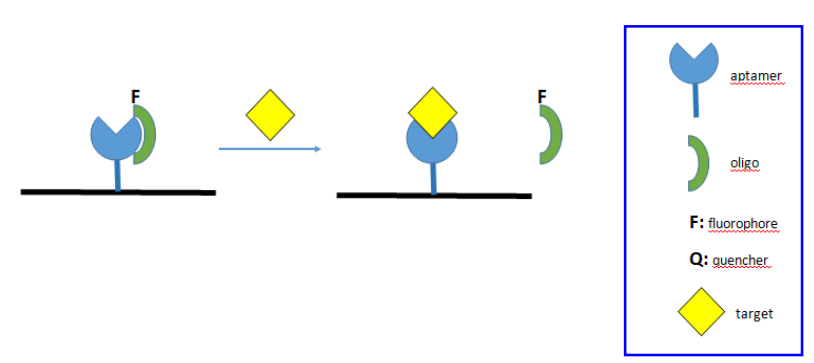

Fig. 3. Fluorescent oligos complementary to aptamers immobilized on the surface of the reaction chamber are displaced by the target molecule.

\section{EXPERIMENTAL RESULTS AND PRELIMIINARY OUTCOMES OF THE RESEARCH}

The Micro-ELISA LOC device and system have been set up to execute both competitive and non-competitive assays, in order to detect small molecules as well as macromolecules. Regarding small molecules, like aflatoxins, a competitive ELISA design is preferred, since it allows the detection of very low concentration of the target. As for macromolecules, like gluten and lysozyme, a non-competitive ELISA design is preferred, in order to reach high specificity. Experiments were carried out to compare standard laboratory ELISA and MicroELISA LOC measurement of different concentrations of target molecules. As molecular model for competitive ELISA assay design, different Aflatoxin B1 concentrations $(0.1 \mathrm{ng} / \mathrm{mL}, 0.6$ $\mathrm{ng} / \mathrm{mL}, 1.2 \mathrm{ng} / \mathrm{mL}$ ) have been tested. As molecular model for non-competitive ELISA assay design, different lysozyme concentrations ( $1.5 \mathrm{ng} / \mathrm{mL}, 6.25 \mathrm{ng} / \mathrm{mL}, 12.5 \mathrm{ng} / \mathrm{mL})$ have been tested. The resulting curves overlapped with standard ELISA, as showed in Figures 4 and 5. 


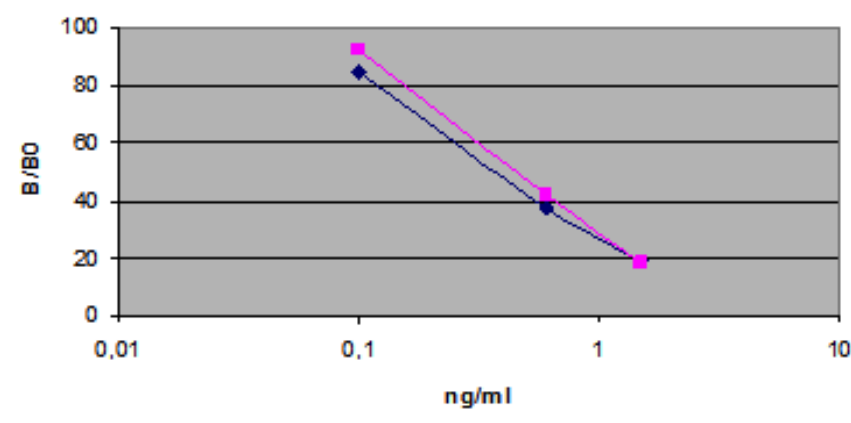

Fig. 4. Comparison between the performances of MicroELISA (blue line) and a laboratory ELISA test for aflatoxin B1 (purple line).

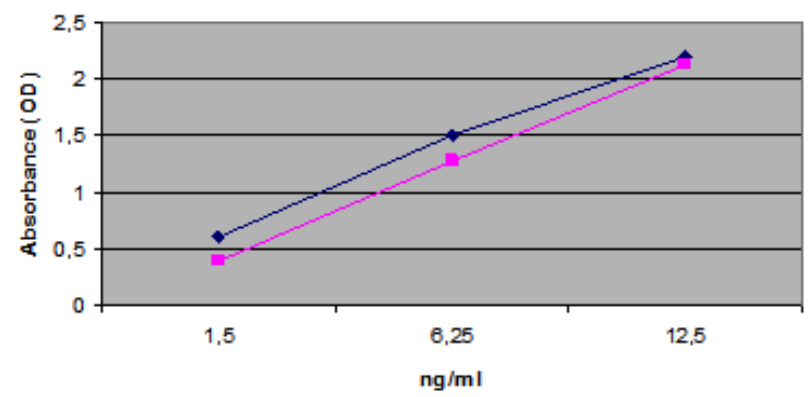

Fig. 5. Comparison between the performances of MicroELISA (blue line) and a laboratory ELISA test for lysozyme (purple line).

In addition, in order to test the reproducibility of analytical results, different experimental sessions have been planned to calculate the coefficient of variation $(\mathrm{CV})$. The $\mathrm{CV}$ is defined as the ratio of the standard deviation $\sigma$ to the mean $\mu$, and it is a very important parameter to evaluate the reliability of the measurements. Usually, for commercial ELISA assays, the $\mathrm{CV}$ is $<10 \%$. The Micro-ELISA LOC device has been optimized to reach this requirement, and the data collected are shown in table 1.

\begin{tabular}{|c|c|}
\hline \multicolumn{2}{|c|}{ Aflatoxin B1 } \\
\hline Session & CV\% \\
\hline 1 & 7 \\
\hline 2 & 8.1 \\
\hline 3 & 8.5 \\
\hline \multicolumn{2}{|c|}{ Lysozime } \\
\hline Session & CV\% \\
\hline 1 & 6.4 \\
\hline 2 & 10 \\
\hline 3 & 9 \\
\hline
\end{tabular}

Tab. 1 Micro-ELISA optimization.
These results are referred to Micro-ELISA LOC devices fabricated by lab-scale prototyping techniques and operatordepended assembly. Thus, the reproducibility of the fabrication and of the bio-functionalization needs to be achieved using industrial grade equipment.

Preliminary outcomes confirm that the developed portable device shows similar test quality compared to conventional laboratory ELISA, opening new interesting challenges in the setting up of detection systems to be exploited during the food supply and production chain.

\section{ACKNOWLEDGMENT}

The work was part of the project "Food Digital Monitoring", financed by the Piedmont Region with European Funds for Regional Development (Call: Smart Factory Platform).

\section{REFERENCES}

[1] Bakeev, Katherine A., ed. Process analytical technology: spectroscopic tools and implementation strategies for the chemical and pharmaceutical industries. John Wiley \& Sons, 2010.

[2] FDA, United States Food and Drug Administration. Guidance for industry: PAT - A framework for innovative pharmaceutical development, manufacturing and quality assurance. Rockville, USA: U.S. Department of Health and Human Services, 2004.

[3] Undey, Cenk, et al., eds. Pat applied in biopharmaceutical process development and manufacturing: an enabling tool for quality-by-design. CRC Press, 2011.

[4] Narsaiah, K., et al. "Optical biosensors for food quality and safety assurance - a review." Journal of food science and technology 49.4 (2012): 383-406.

[5] Thakur, M. S., and K. V. Ragavan. "Biosensors in food processing." Journal of food science and technology 50.4 (2013): 625-641.

[6] Council for Agricultural Science and Technology. Mycotoxins: Economic and Health Risks; CAST: Ames, IA, USA, 1989; p.99

[7] Kensler, Thomas W., et al. "Aflatoxin: a 50-year odyssey of mechanistic and translational toxicology." Toxicological Sciences 120.suppl_1 (2010): S28-S48.

[8] Binder, E. M., et al. "Worldwide occurrence of mycotoxins in commodities, feeds and feed ingredients." Animal feed science and technology 137.3 (2007): 265-282.

[9] Liu, Yan, and Felicia Wu. "Global burden of aflatoxin-induced hepatocellular carcinoma: a risk assessment." Environmental health perspectives 118.6 (2010): 818.

[10] Kim, Sung Gun, et al. "Ultrasensitive Bisphenol A Field-Effect Transistor Sensor Using an Aptamer-Modified Multichannel Carbon Nanofiber Transducer." ACS applied materials \& interfaces 8.10 (2016): 6602-6610.

[11] Dong, Yiyang, et al. "Aptamer and its potential applications for food safety." Critical reviews in food science and nutrition 54.12 (2014): 1548-1561.

[12] Bhardwaj and McGoron. "Biosensor Technology for Chemical and Biological Toxins: Progress and Prospects". Photon Journal of Biomedical Engineering 112 (2014) 380-392 